\title{
No Longer Marketed
}

National Cancer Institute

\section{Source}

National Cancer Institute. No Longer Marketed. NCI Thesaurus. Code C101675.

Indicates that a product was previously promoted for use, but currently is not being promoted. 